\title{
IFNT-independent effects of intrauterine extracellular vesicles (EVs) in cattle
}

\author{
Keigo Nakamura ${ }^{1}$ Kazuya Kusama ${ }^{1,2}$, Atsushi Ideta ${ }^{3}$, Kazuhiko Imakawa ${ }^{4}$ and Masatoshi Hori ${ }^{1}$ \\ ${ }^{1}$ Laboratory of Veterinary Pharmacology, Graduate School of Agricultural and Life Sciences, The University of Tokyo, \\ Bunkyo, Tokyo, Japan, ${ }^{2}$ Department of Endocrine Pharmacology, Tokyo University of Pharmacy and Life Sciences, \\ Hachioji, Tokyo, Japan, ${ }^{3}$ Zen-noh Embryo Transfer Center, Fukuoka, Fukuoka, Japan and ${ }^{4}$ Research Institute of \\ Agriculture, Tokai University, Kumamoto, Kumamoto, Japan
}

Correspondence should be addressed to K Kusama; Email: kusamak@toyaku.ac.jp

\begin{abstract}
Extracellular vesicles (EVs) present in uterine lumen are involved in conceptus-endometrial interactions during the pre-implantation period. Despite numerous studies conducted on interferon tau (IFNT), a major protein of maternal recognition of pregnancy, the effect of intrauterine EVs on the endometrium during pre-implantation periods has not been well-characterized. To characterize conceptus-derived intrauterine EVs independent of IFNT, transcripts found from RNA-seq analysis in RNAs extracted from primary bovine endometrial epithelial cells (EECs) treated with cyclic day 17 (C17) EVs, pregnant day 17 (P17) EVs or IFNT were analyzed. These analyses identified 82 transcripts uniquely induced by IFNT-independent P17 EVs, of which a large number of transcripts were associated with 'the TNF signaling pathway' and 'Inflammatory response'. Moreover, high expression of CD40L, a member of the TNF superfamily, and its receptor CD40 were found in P17 EVs and in EECs, respectively. Furthermore, the expression of TNF signaling pathway-related genes was up-regulated by the treatment with P17 EVs, but these increases were down-regulated by NF-kB signaling inhibitor. These findings suggest that P17 EVs could induce a pro-inflammatory response in the endometrium, independent of IFNT, to regulate uterine receptivity, facilitating conceptus implantation.

Reproduction (2020) 159 503-511
\end{abstract}

\section{Introduction}

Approximately half of embryonic losses are known to occur prior to the time of maternal recognition of pregnancy, which is around day 16 in cattle, during the critical period for corpus luteum maintenance or demise (Wiltbank et al. 2016). Over the past decade, various global analyses were implemented to study the expression of transcripts in conceptus or endometrium, identifying many molecules with potential importance during the implantation period (Mamo et al. 2011, Forde et al. 2014, 2015, Bai et al. 2018). Although data continue to accumulate, mechanisms by which these factors function during the conceptus attachment process remain unclear.

In cattle, endometrial gene expression between cyclic and pregnant animals did not differ until pregnant days 15-17 (Walker et al. 2010, Forde et al. 2011, Bauersachs et al. 2012), but transcriptomic differences between cyclic and pregnant animals become apparent at the time of pregnancy recognition. Interferon tau (IFNT) is the major factor essential for the process of maternal recognition of pregnancy (Bazer \& Thatcher 2017, Ealy \& Wooldridge 2017, Forde \& Lonergan 2017, Roberts 2017). It has been reported that sufficient concentrations of IFNT must be released by the elongating conceptus between pregnant days 15 and 17 (P15 and P17, respectively), inducing the expression of a large number of interferon-stimulated genes in the endometrium, which leads to the establishment of uterine receptivity to conceptus implantation in ruminant ungulates (Spencer et al. 2013, Wiltbank et al. 2016). In spite of extensive data accumulation, pregnancy rate in cattle remains relatively low due to early embryonic losses. These observations indicate that molecular mechanisms or factors in the endometrium which play a major role along with IFNT in pregnancy establishment periods need to be elucidated.

Different cell types generally interact with secretory soluble factors such as hormones and cytokines. In recent years, however, the role that extracellular vesicles (EVs) play in cell-cell interaction has been well-documented. EVs contain lipids, proteins and nucleic acids, and specific target cells have surface receptors, with which ligands from the originating cells have the potential to selectively interact (Burns et al. 2018). Evidence has been accumulated that EVs are present in most of bodily fluids, including uterine flushing fluids (UFs) (Piehl et al. 2013, Prunotto et al. 2013, Cleys et al. 2014, Nguyen et al. 2016). In ruminants, UFs components 
have been reported to induce the conceptus elongation and attachment (Kusama et al. 2016, Simintiras et al. $2019 a, b, c)$. It was also reported that EVs released by conceptuses and/or endometrium during the periimplantation period are involved in biochemical and/ or physical interactions between the conceptus and the endometrium (Burns et al. 2014, Ruiz-González et al. 2015, Burns et al. 2016, Nakamura et al. 2016, Kusama et al. 2018a).

Based on these findings, we hypothesized that in addition to IFNT, intrauterine EVs on P17 are essential for the process of maternal recognition of pregnancy, which regulates the uterine environment, facilitating subsequent conceptus implantation to the uterine epithelium. Using RNA-seq analysis in this study, we first evaluated effects of cyclic day 17 (C17) and P17 $\mathrm{EV}$ s on the primary bovine endometrial epithelial cells (EECs), from which pro-inflammatory response-related IFNT-independent factors were further evaluated.

\section{Materials and methods \\ Collection of bovine uterine flushing fluids}

All animal procedures in this study were performed in accordance with the guidelines of the Committee for Experimental Animals at Zen-noh Embryo Transfer Center, and approval was also obtained from the Ethics Committee of the University of Tokyo (IRB number 7A-6-605). Estrous synchronization, superovulation, and embryo transfer (ET) processes were performed as previously described (Ideta et al. 2007). Recipient heifers were given a single injection of $0.75 \mathrm{mg}$ cloprostenol to synchronize their estrous cycles. The day of injection was considered day 0 of estrus. For ET processes, day 7 embryos were collected from super-ovulated and artificially inseminated (AI) Japanese black cattle. Two blastocysts each derived from the superovulation/Al procedure were then transferred non-surgically into the uterine horn of Holstein heifers, ipsilateral to the corpus luteum, on day 7 of the estrous cycle. Elongated conceptuses on day 17 were then collected non-surgically by uterine flushing with $500 \mathrm{ml}$ sterile PBS ( $\mathrm{pH} 7.2)$. Conceptuses in the UF media were obtained by centrifugation at $1000 \mathrm{~g}$ for $5 \mathrm{~min}$ and snap-frozen in liquid nitrogen. Following conceptus removal, the media and those from day 17 cyclic animals were centrifuged at $4000 \mathbf{g}$ for 5 min to remove cell debris, supernatants were filtered through $0.22 \mathrm{~nm}$ membrane, and then samples were stored at $-80^{\circ} \mathrm{C}$ until use.

\section{Isolation of EVs from uterine flushing fluids}

EVs were isolated from $\mathrm{C} 17$ and P17 UFs by adding exosome precipitation solution (Exo-Quick-TC, System Biosciences, Mountain View, CA, USA) according to the manufacturer's instructions. The UFs with Exo-Quick-TC were incubated overnight at $4^{\circ} \mathrm{C}$ and then centrifuged at $1500 \mathrm{~g}$ for $30 \mathrm{~min}$ at $4^{\circ} \mathrm{C}$ to pellet EVs. EVs were suspended and their protein concentrations adjusted either in PBS $(1 \mathrm{mg} / \mathrm{mL})$ for in vitro culture experimentations or in mammalian protein extraction reagent (M-PER, Thermo Fisher Scientific) for Western blot analysis (Nakamura et al. 2016, Kusama et al. 2018a).

\section{Transmission electron microscopy}

The EVs in PBS were placed on a carbon-film grid and were partially dried. The staining solution of $2 \%$ uranyl acetate in water was added to the grids for 2 min and the excess liquid was blotted off with filter paper, followed by overnight drying at room temperature. The grids were analyzed through the use of a HITACHI H-7600 Transmission Electron Microscope (TEM, Hitachi High-Technologies Corporation, Tokyo, Japan) at Hanaichi UltraStructure Research Institute (Aichi, Japan).

\section{Nanoparticle tracking analysis}

The EVs isolated from $\mathrm{C} 17$ or P17 UFs and suspended in PBS (2-6 $\times 10^{8}$ particles $\left./ \mathrm{ml}\right)$ were subjected to nanoparticle tracking analysis using a NanoSight NS300 (NanoSight Ltd, Amesbury, UK) instrument with $488 \mathrm{~nm}$ laser and a complementary metal-oxide-semiconductor camera (Andor Technology, Belfast, UK) and NanoSight NTA 3.2 software calibrated with $100 \mathrm{~nm}$ polystyrene beads (Thermo Fisher Scientific). Videos were recorded for $30 \mathrm{~s}$ during which the nanoparticle tracking analysis software (NanoSight Ltd) tracked each visible particle. The Stokes-Einstein equation was employed to determine the size distribution and number of particles within the sample (Nakamura et al. 2016).

\section{Cell preparation and culture conditions}

Isolation and culture of EECs were carried out as previously described (Sakurai et al. 2012). In brief, uteri of healthy Holstein cows were obtained from a local abattoir in accordance with protocols approved by the local Institutional Animal Care, Use and Ethics Committee at Okayama University, Okayama, Japan. Uteri of the early luteal phase (days 2-5) were excised and immediately transported to the laboratory. The uterine lumen was trypsinized $(0.3 \% \mathrm{w} / \mathrm{v})$, from which EECs were isolated. EECs were then cultured on collagen type IA-coated plates in Dulbecco modified Eagle medium/F12 (DMEM/F12) (1:1) medium (Wako Pure Chemical Industries) supplemented with $10 \%(\mathrm{v} / \mathrm{v})$ newborn calf serum (Thermo Fisher Scientific), $2 \mathrm{mM}$ glutamine (Thermo Fisher Scientific), and antibiotic/ antimycotic solution (Thermo Fisher Scientific) at $37^{\circ} \mathrm{C}$ under $5 \% \mathrm{CO}_{2}$ in humidified air. EECs $\left(5 \times 10^{4}\right.$ cells/well $)$ placed onto collagen type IA-coated 12-well plate were further incubated with or without $\mathrm{C} 17$ or P17 EVs $(10 \mu \mathrm{g} /$ well $)$ in serum-free DMEM/F12 for $48 \mathrm{~h}$. For the inhibition of NF-kB pathway, EECs $\left(5 \times 10^{4}\right.$ cells/well) were cultured in 12 -well plate with or without P17 EVs $(10 \mu \mathrm{g} /$ well $)$ and/or the selective IkB kinase inhibitor, NF-kB pathway inhibitor, 2-((aminocarbonyl)amino)5-(4-fluorophrnyl)-3-thiophenecarboxaminde (TPCA1) (20 $\mathrm{nM}$, ab145522, Abcam) for $48 \mathrm{~h}$.

\section{RNA extraction and quantitative $R T-P C R$}

Using the ISOGEN reagent (Nippon Gene, Tokyo, Japan), total RNAs were extracted from cultured EECs according 
to the manufacturer's protocols. The isolated RNA was reverse-transcribed to cDNA using ReverTra Ace gPCR RT Kit (Toyobo, Osaka, Japan), which was then subjected to qPCR amplification using PowerUP SYBR Green Master Mix (Thermo Fisher Scientific). All primers are listed in Table 1. The qPCR amplification was carried out on an Applied Biosystems STEP One Plus real-time PCR System (Applied Biosystems). Amplification efficiencies of all target genes and the reference genes, glyceraldehyde-3-phosphate dehydrogenase (GAPDH) and actin beta (ACTB), were examined through their calibration curves and found to be comparable. Average threshold $(\mathrm{Ct})$ values for each target were determined by Sequence Detection System software v2.3 (Applied Biosystems) (Nakamura et al. 2017).

\section{RNA sequencing, data, gene ontology, and pathway analyses}

Total RNA for RNA-seq analysis was extracted from cultured EECs using Isogen (Nippon gene) according to the manufacturer's instructions. High-throughput sequencing libraries were prepared using the TruSeq Stranded mRNA LT Sample Prep Kit (Illumina, San Diego, CA, USA) according to the manufacturer's instructions, and the analysis was performed by Macrogen Japan (Kyoto, Japan). Primary sequencing data were deposited to the DDBJ (DNA Data Bank of Japan) Sequence Read Archive (https://www.ddbj.nig.ac.jp/dra/index-e.html) (accession numbers DRR174782 to DRR174790). Data analysis was performed as described previously (Kusama et al. 2017). Briefly, trimmed sequences were analyzed on the basis of the TopHat/Cufflinks pipeline based on the bovine genome (bosTau8) and reference annotations obtained from
UCSC genome browser (https://genome.ucsc.edu). Differential and significant gene expression analysis was performed with the use of FPKM (fragments per kilo-base of gene locus summarized mRNA per million reads). Genes were selected with the criteria of an absolute expression level $>1$ FPKM. The gene ontology (GO) and enriched signaling pathway analyses were performed with the Enrichr tool (http://amp.pharm.mssm. edu/Enrichr/). RNA-seq data sets obtained were compared with previously published data from culture endometrial explants treated with or without ovine IFNT or a day 15 Al-derived conceptus (Mathew et al. 2019, Sánchez et al. 2019) and those from IFNT-treated EECs (DRR083698 to DRR083699) (Kusama et al. 2017).

\section{Western blot analysis}

EVs and EECs lysed with M-PER $(10 \mu \mathrm{g})$ were separated through SDS-PAGE and were then transferred onto polyvinylidene difluoride (PVDF) membranes (Bio-Rad). After blocking with Block Ace reagent (DS Pharma Biomedical, Osaka, Japan), membranes were incubated with rabbit polyclonal antihuman CD63 antibody (1:2000, EXOAB-CD63A-1, System Biosciences), rabbit polyclonal anti-human HSP70 antibody (1:2000, EXOAB-HSP70A-1, System Biosciences), rabbit polyclonal anti-human CD40L (1:100, ARP33831_P050, Aviva Systems Biology, San Diego, CA, USA), rabbit polyclonal anti-bovine TNFA (1:1000, GTX38917, GeneTex, Irvine, CA, USA), rabbit monoclonal anti-human NF-kB p65 (C22B4, 1:1000, Cell signaling Technology), rabbit polyclonal antihuman NF-kB p65 (acetyl K310) (1:1000, ab19870, Abcam), or rabbit polyclonal anti-human ACTB (1:5000, ab1801, Abcam). Immunoreactive bands were detected using enhanced

Table 1 Primers for real-time PCR analyses.

\begin{tabular}{|c|c|c|}
\hline Name (Accession No.) & Sequence & Product length $(\mathrm{bp})$ \\
\hline \multirow[t]{2}{*}{ BIRC3 (NM_001035293) } & F: 5'-TCCAGATGTGGCCGTTGACT-3' & 129 \\
\hline & R: 5'-CCTTCGGTTCCCAATTGCTC-3' & \\
\hline \multirow[t]{2}{*}{ CD40 (NM_001105611) } & F: 5'-CGGTAAAGGCGAATTCTTGTCC-3' & 139 \\
\hline & R: 5'-GCCTTCGACACATACACAAGTG-3' & \\
\hline \multirow[t]{2}{*}{ CSF2 (NM_174027) } & F: 5'-GAATGACACAGAAGTCGTCTCTG-3' & 120 \\
\hline & R: 5'-AAGGAGCCCATGAGACTAGTG-3' & \\
\hline \multirow[t]{2}{*}{ CXCL2 (NM_174299) } & F: 5'-AGAAGCTCTTGGATGGCTGTTCCA-3' & 91 \\
\hline & R: 5'-AGATGGCCTTAGGAGGTGGTGATT-3' & \\
\hline \multirow[t]{2}{*}{ CXCL3 (NM_001046513) } & F: 5'-ACTGTGGCCAAACCGAAGTC-3' & 118 \\
\hline & R: 5'-TTGGTGCTGCCCTTGTTTAG-3' & \\
\hline \multirow[t]{2}{*}{ CXCL5 (NM_174300) } & F: 5'-TCGCCACTATGAGACTGCTATC-3' & 150 \\
\hline & R: 5'-AACGCAGCTCTCTCACAACG-3' & \\
\hline \multirow[t]{2}{*}{ IL6 (NM_173923) } & F: 5'-ACCGAAGCTCTCATTAAGCG-3' & 105 \\
\hline & R: 5'-TTCTGCCAGTGTCTCCTTGC-3' & \\
\hline \multirow[t]{2}{*}{ MMP9 (NM_174744) } & F: 5'-CCCGGATCAAGGATACAGCC-3' & 177 \\
\hline & R: 5'-GGGCGAGGACCATACAGATG-3' & \\
\hline \multirow[t]{2}{*}{ NFKBIA (NM_001045868) } & F: 5'-TCCTGCACTTAGCCATCATCC-3' & 145 \\
\hline & R: 5'-TCTGGCTGGTTAGTGATCACAG-3' & \\
\hline \multirow[t]{2}{*}{ TNFAIP3 (NM_001192170) } & F: 5'-TTGCAACATCCTCAGAAGGC-3' & 101 \\
\hline & R: 5'-AAATCCCACCCACCTTCAGAG-3' & \\
\hline \multirow[t]{2}{*}{ ACTB (NM_173979) } & F: 5'-ATATTGCTGCGCTCGTGGTTG-3' & 148 \\
\hline & R: 5'-TAGGAGTCCTTCTGGCCCATG-3' & \\
\hline \multirow[t]{2}{*}{ GAPDH (NM_001034034) } & F: 5'-GCATCCCTGAGACAAGATGGTG-3' & 113 \\
\hline & R: 5'-CATTGATGGCAACGATGTCCAC-3' & \\
\hline
\end{tabular}

F: Forward; R: Reverse. 
chemiluminescence (EMD Millipore) after incubation with horseradish peroxidase labeled goat anti-rabbit IgG (1:5000, Vector Laboratories, Burlingame, CA, USA). Signals were detected using C-DiGit Blot Scanner (LI-COR) and then band density was assessed with Image Studio DiGit software (version 5.2) (Kusama et al. 2018b).

\section{Statistical analysis}

All experimental data from qPCR analyses represent the results obtained from three or more independent experiments, each with triplicate assays. Data were expressed as the mean \pm S.E.M. Significance was assessed using the Tukey-Kramer multiple comparisons test. A P-value of $<0.05$ was considered statistically significant.

\section{Results}

\section{EVs present in bovine UFs on cyclic and pregnant days 17}

To characterize EVs in bovine UFs based on the extracellular vesicles 2018 (MISEV2018) guidelines (Théry et al. 2018), EV markers, CD63 and HSP70, were detected in C17 and P17 UFs (Fig. 1A). Moreover, TEM detected vesicles of 50-150 nm in diameter in the isolated pellets from C17 and P17 UFs (Fig. 1B), and C17 and P17 EVs were subjected to nanoparticle tracking analysis, revealing 116.4 (mean) $\pm 50.2 \mathrm{~nm}$ (S.D.) and $108.9 \pm 39.4 \mathrm{~nm}$, respectively (Fig. 1C). These results indicated that $\mathrm{EVs}$ were secreted into the uterine lumen during pre-implantation periods.

A
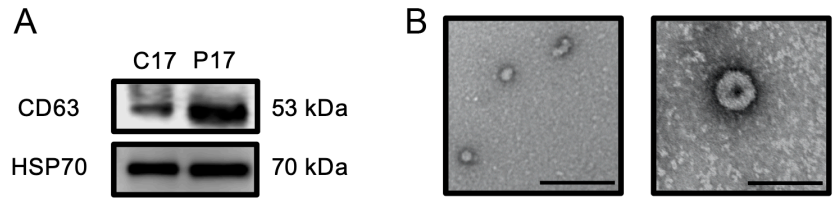

\section{Transcript changes in EECs treated with intrauterine EVs from day 17 cyclic animals}

To examine effects of $\mathrm{C} 17 \mathrm{EVs}$ derived from bovine endometrium on gene expression in EECs, RNA-seq analysis was executed, identifying 9164 transcripts, from which eight genes were up-regulated and 11 genes were down-regulated (Fig. 2A). A pair plot comparison of C17 EVs treated and EECs without any treatment (control groups) showed that there were very few differentially expressed genes (DEGs) (Fig. 2B).

\section{IFNT-independent effects of P17 EVs in EECs}

Along with others, our group previously indicated that conceptus-derived EVs were present in UFs, which could function to modify endometrial response
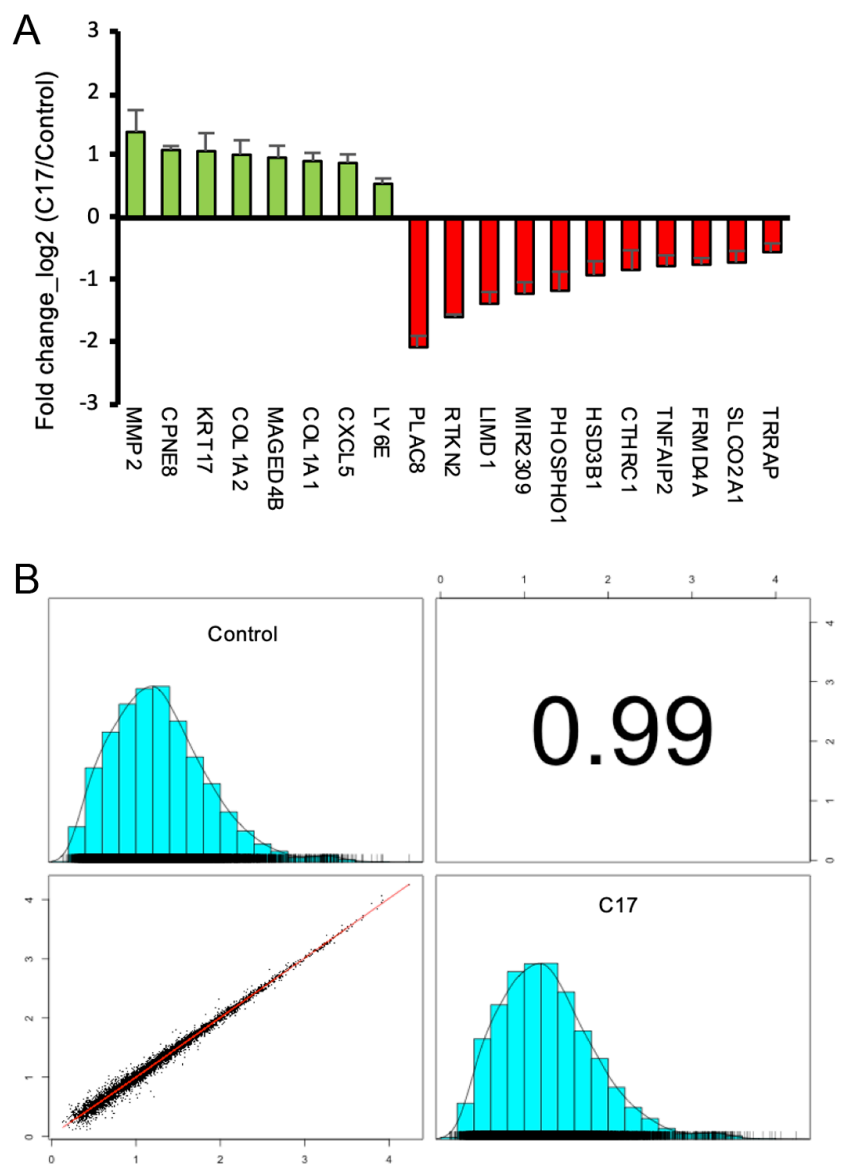

Figure 2 Transcript changes in EECs treated with intrauterine EVs on cyclic day 17. RNAs were extracted from primary bovine endometrial epithelial cells (EECs) incubated without (control) or with C17 EVs $(10 \mu \mathrm{g})$ for $48 \mathrm{~h}$ and then were subjected to RNA-seq analysis. Data were from three independent in vitro culture experiments. (A) Fold changes in 19 differentially expressed genes (DEGs) with q-values less than 0.05 and 2 -fold changes identified by RNA-seq were shown. (B) These diagrams show pair plots comparison between control and C17 EVs-treatment groups and density plots in each group. Figure shows the correlation coefficient between control and C17 EVs.
Figure 1 Characterization of EVs isolated from UFs during the pre-implantation period. (A) Western blot analysis showed the presence of CD63 and HSP70 in pellets isolated from C17 or P17 bovine UFs. (B) Transmission electron microscopy analysis revealed the presence of 50-150 nm vesicles in UFs, consistent with those of EVs. Scale bar $=400 \mathrm{~nm}$ (left) and $200 \mathrm{~nm}$ (right). (C) Nanoparticle tracking analysis of $\mathrm{C} 17$ or $\mathrm{P} 17$ revealed that the range of vesicles size is $50-200 \mathrm{~nm}$, respectively. 
(Burns et al. 2016, Kusama et al. 2017). To characterize the transcripts induced by conceptus-derived molecules in detail, RNA-seq analysis of EECs treated with P17 EVs was executed and the DEGs with 2.0-fold changes were then compared among C17 EVs, P17 EVs, and previously reported conceptuses treatment groups (Mathew et al. 2019, Sánchez et al. 2019). Forty-one DEGs found in common between P17 EVs and conceptuses treatment groups indicated that these DEGs were regulated by conceptus-derived EVs (Fig. 3A). Pathway and GO analyses were then executed to identify conceptusderived DEGs' significantly enriched pathways and biological process terms, from which the most enriched were associated with 'Interferon signaling' and 'Cellular response to type I interferon' respectively (Fig. 3B).

Although it was reported that bovine conceptus induces the IFNT-independent expression of endometrial transcriptomes during the pre-attachment period (Mathew et al. 2019), their molecular mechanisms have not been characterized. To test the hypothesis that IFNT-independent endometrial transcriptome was regulated by intrauterine EVs, in addition to $\mathrm{C} 17$ and P17 EVs-treatment groups, the effect of IFNT on the gene expression in EECs previously demonstrated (Kusama et al. 2017) was compared. The venn diagram shows the number of DEGs with 2.0-fold changes among C17 EVs, P17 EVs, and IFNT treatment groups compared with those of the control group. Of these 114 DEGs, 82 transcripts were expressed in only the P17 EVs-treatment group, but not the $\mathrm{C} 17 \mathrm{EV}$ s or the IFNT-treated group (Fig. 3C). IFNT-independent DEGs induced by P17 EVs were then subjected to enrichment pathway and GO analyses. These analyses revealed significantly enriched pathways and biological process terms, from which the most enriched were 'The tumor necrosis factor (TNF) signaling pathway' and 'Inflammatory response' (Fig. 3D). The details of these analyses are summarized in Tables 2 and 3. Furthermore, qPCR analysis ascertained that changes in TNF signaling pathway-related genes, CXCL2, CXCL3, CXCL5, CSF2, NFKBIA, TNFAIP3, IL6, $B I R C 3$ and MMP9, were similar to those of the RNA-seq analysis (Fig. 3E).

\section{Effect of exosomal CD40L on the activation of NF-kB in EECs}

RNA-seq analysis and qPCR in this study revealed that along with TNF signaling pathway-related genes, CD40, a member of the TNF receptor superfamily, was up-regulated by the treatment of P17 EVs (Fig. 4A). Furthermore, CD40 ligand (CD40L) protein expression was higher in P17 intrauterine EVs than that of C17 (Fig. 4B), whereas TNFA was not detected (result not shown). A previous study reported that the binding CD40L to CD40 leads to activation of NF-kB signaling (Hostager \& Bishop 2013). We therefore investigated whether P17 $\mathrm{EVs}$ induced the activation of NF-kB in EECs, resulting
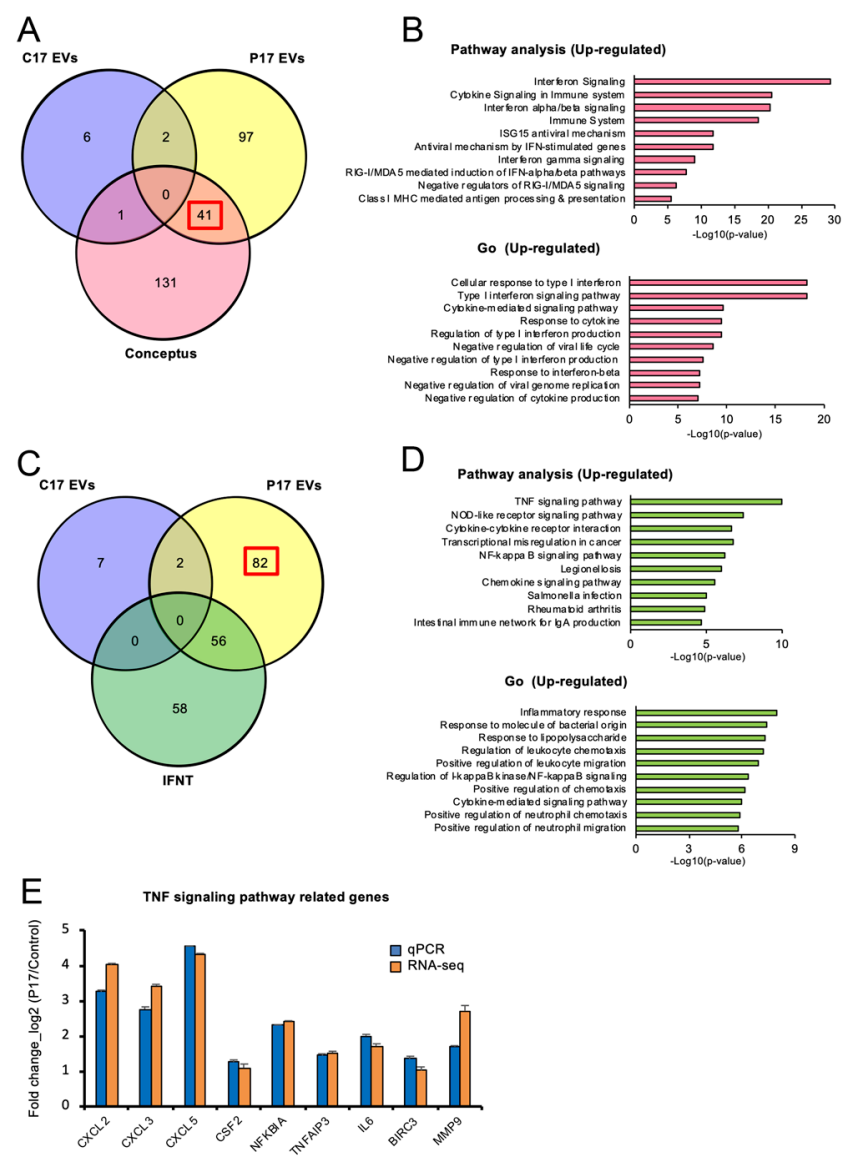

Figure 3 IFNT-independent effects of P17 EVs in EECs. (A) Venn diagram shows the number of genes with 2.0-fold changes among $\mathrm{C} 17 \mathrm{EVs}, \mathrm{P} 17 \mathrm{EVs}$, and conceptuses treatment groups compared with control group, from which 41 transcripts (red rectangle) were identified in common between P17 EVs and conceptuses treatment groups. (B) Up-regulated conceptus-derived DEGs in EECs treated with P17 EVs were functionally classified by the biological process in GO terms and enriched pathway analyses. (C) Venn diagram shows the number of genes with 2.0-fold changes among C17 EVs, P17 EVs, and IFNT treatment groups compared with control group, from which 82 transcripts (red rectangle) were identified in only P17 EVstreatment group. (D) Up-regulated IFNT-independent DEGs in EECs treated with P17 EVs were functionally classified by the biological process in GO terms and enriched pathway analyses. (E) Fold changes in TNF signaling pathway-related genes in EECs incubated without (control) or with P17 EVs $(10 \mu \mathrm{g})$ for $48 \mathrm{~h}(n=3$ each) were examined by qPCR or RNA-seq. ACTB and GAPDH mRNA served as internal controls for RNA integrity.

that the P17 EVs increased acetylated NF-kB, an active NF-kB, in EECs (Fig. 4C). To further examine whether P17 EVs containing CD40L up-regulated the TNF signaling pathway-related gene expression through the activation of NF-kB signaling, EECs were treated with the P17 EVs in combination with or without NF-kB pathway inhibitor TCPA1. Treatment of TCPA1 decreased the expression of CXCL2, CXCL3, CXCL5, CSF2, NFKBIA, TNFAIP3, IL6, $B I R C 3$, and MMP9, all of which were induced by P17 EVs (Fig. 4D). 
Table 2 Gene expression related to P17 EVs-induced enriched pathways, which are independent of IFNT.

\begin{tabular}{|c|c|c|}
\hline Enrichment pathway & $P$-value & Genes \\
\hline \multicolumn{3}{|l|}{ Up-regulation } \\
\hline TNF signaling pathway & $1.08 \mathrm{E}-10$ & NFKBIA, IL6, CSF2, TNFAIP3, CXCL3, MMP9, CXCL2, CXCL5, BIRC3 \\
\hline NOD-like receptor signaling pathway & 3.57E-08 & NFKBIA, IL6, CXCL8, TNFAIP3, CXCL2, BIRC3 \\
\hline Cytokine-cytokine receptor interaction & $2.40 \mathrm{E}-07$ & IL22RA1, CD40, IL6, CSF2, CXCL8, CXCR4, CXCL3, CXCL2, CXCL5 \\
\hline Transcriptional misregulation in cancer & $1.53 \mathrm{E}-07$ & CD4O, IL6, CSF2, CXCL8, NFKBIZ, PLAT, MMP9, BIRC3 \\
\hline NF-kappa B signaling pathway & $6.82 \mathrm{E}-07$ & NFKBIA, CD40, CXCL8, TNFAIP3, CXCL2, BIRC3 \\
\hline Legionellosis & $1.11 \mathrm{E}-06$ & NFKBIA, IL6, CXCL8, CXCL3, CXCL2 \\
\hline Chemokine signaling pathway & $3.02 \mathrm{E}-06$ & NFKBIA, CXCL8, CXCR4, ARRB1, CXCL3, CXCL2, CXCL5 \\
\hline Salmonella infection & $1.02 \mathrm{E}-05$ & IL6, CSF2, CXCL8, CXCL3, CXCL2 \\
\hline Rheumatoid arthritis & $1.28 \mathrm{E}-05$ & IL6, CSF2, CXCL8, MMP1, CXCL5 \\
\hline Intestinal immune network for IgA production & 2.00E-05 & PIGR, CD40, IL6, CXCR4 \\
\hline
\end{tabular}

\section{Discussion}

This study provides the first evidence that intrauterine EVs-induced IFNT-independent modifications to the bovine endometrial transcriptome. Although several studies have shown the presence and potential function of the EVs and EVs containing IFNT during the implantation period in ruminants (Burns et al. 2016, Mellisho et al. 2017), their IFNT-independent effects on EECs have not been characterized. In this study, IFNT-independent P17 EVs, but not C17 EVs, induced 82 transcripts, and a large number of transcripts were associated with 'the TNF signaling pathway' and 'Inflammatory response'. Moreover, high expression of CD40L, a member of TNF superfamily, and its receptor CD40 were found in P17 EVs and in EECs, respectively. Furthermore, up-regulation of TNF signaling pathwayrelated genes by treatment with $\mathrm{P} 17 \mathrm{EVs}$ was downregulated by NF-kB signaling inhibitor, suggesting that P17 EVs, independent of IFNT, induced TNF signaling pathway-related genes via CD40L/CD40/NF-kB pathway in primary bovine endometrial epithelial cells.

IFNT is the main trophectodermal factor which acts on uterine endometrium and attenuates luteolytic mechanisms, leading to the continued production of progesterone. Our previous study showed that P17, P20, and P22 EVs contained IFNT and that the expression of IFN-stimulated genes (ISGs) such as ISG15, MX1, MX2, STAT1, and STAT2 in EECs was up-regulated in response to the these EVs (Kusama et al. 2018a). To investigate effects of conceptus-derived EVs, the DEGs in uterine endometrium were compared among C17 EVs, P17 EVs, and conceptuses treatment groups (Mathew et al. 2019, Sánchez et al. 2019). 41 DEGs were found as common factors between P17 EVs and conceptuses treatment, which were associated with 'interferon signaling' and 'Cellular response to type I interferon' in significantly enriched pathways and biological process terms, respectively. These findings were consistent with those from our previous ovine and bovine studies (Nakamura et al. 2016, Kusama et al. 2018a) and supported the notion that EVs containing IFNT secreted from elongated conceptus acts on the endometrial epithelium during the peri-implantation period.

RNA-seq analysis identified 9164 transcripts in EECs treated with $\mathrm{C} 17 \mathrm{EVs}$, from which eight genes, MMP2, CPNE8, KRT17, COL1A2, MAGE4B, COLIA1, CXCL5, and $L Y 6 E$, were up-regulated and 11 genes, PLAC8, RTKN2, LIMD1, MIR2309, PHOSPHO1, HSD3B1, CTHRC1, TNFAIP2, FRMD4A, SLCO2A1, and TRRAP, were down-regulated compared with the control. There were very few DEGs in C17 EVs-treated EECs: the correlation coefficient with control EECs was 0.99; however, of these DEGs, factors that may govern uterine environment required for conceptus implantation were observed. For example, one of the new IFNT-regulated genes with various potential roles such as immunity, differentiation, and proliferation was up-regulated in pregnant cows (Mansouri-Attia et al. 2009,

Table 3 Gene expression related to P17 EVs-induced GO terms, which are independent of IFNT.

\begin{tabular}{|c|c|c|}
\hline GO_BP Term & $P$-value & Genes \\
\hline \multicolumn{3}{|l|}{ Up-regulation } \\
\hline Inflammatory response & $1.16 \mathrm{E}-08$ & PTGFR, CD40, IL6, NCR3, CXCL8, CXCR4, CXCL3, CXCL2, CXCL5, APOL3 \\
\hline Response to molecule of bacterial origin & 4.01E-08 & CD40, IL6, CXCL8, TNFAIP3, CXCL3, CXCL2, CXCL5 \\
\hline Response to lipopolysaccharide & $5.05 \mathrm{E}-08$ & CD40, IL6, CXCL8, ZC3H12A, TNFAIP3, CXCL3, CXCL2, CXCL5 \\
\hline Regulation of leukocyte chemotaxis & $5.76 \mathrm{E}-08$ & CD4O, IL6, CXCL8, ZC3H12A, TNFAIP3, CXCL3, CXCL2, CXCL5 \\
\hline Positive regulation of leukocyte migration & $1.09 \mathrm{E}-07$ & CXCL5, CXCL3, CXCL2, NFKBIA, CXCL8, CD4O \\
\hline Regulation of I-kappaB kinase/NF-kappaB signaling & 4.13E-07 & NFKBIA, CD4O, ECM1, ZC3H12A, TNFAIP3, APOL3, BIRC3, LTF \\
\hline Positive regulation of chemotaxis & $6.17 \mathrm{E}-07$ & IL6, CXCL8, CXCL3, CXCL2, CXCL5 \\
\hline Cytokine-mediated signaling pathway & $1.13 \mathrm{E}-06$ & $\begin{array}{l}\text { IL22RA1, NFKBIA, CD40, IL6, CSF2, CXCL8, MMP1, CXCL3, MMP9, } \\
\text { CXCL2, CXCL5, BIRC3 }\end{array}$ \\
\hline Positive regulation of neutrophil chemotaxis & $1.38 \mathrm{E}-06$ & CXCL8, CXCL3, CXCL2, CXCL5 \\
\hline Positive regulation of neutrophil migration & $1.63 \mathrm{E}-06$ & CXCL8, CXCL3, CXCL2, CXCL5 \\
\hline
\end{tabular}



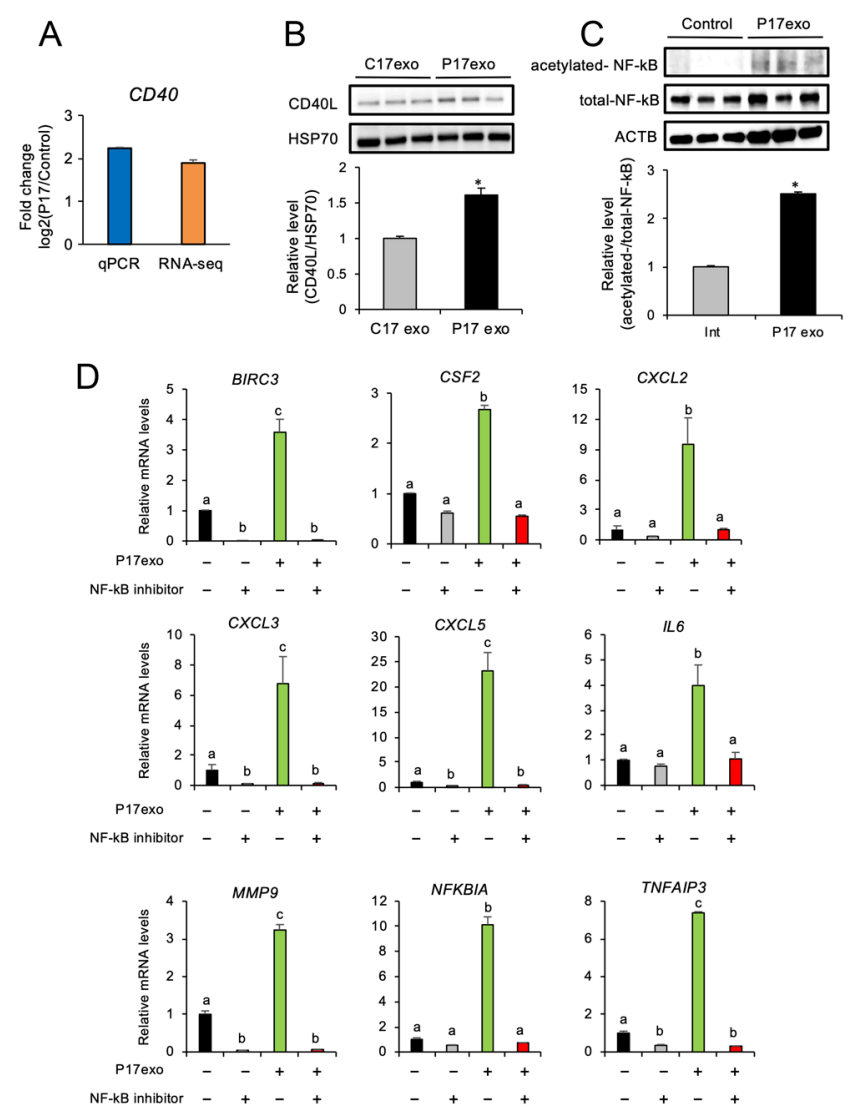

Figure 4 Effect of exosomal CD40L on the activation of NF-kB in EECs. (A) Fold changes in CD4O in EECs incubated without (control) or with P17 EVs $(10 \mu \mathrm{g})$ for $48 \mathrm{~h}(n=3$ each), which were subjected to qPCR or RNA-seq analysis. ACTB and GAPDH mRNA served as internal controls for RNA integrity. (B) The lysates of C17 and P17 EVs were subjected to Western blotting. The bar graphs show CD40L levels normalized by HSP70 levels ( $n=3$ each). $* P<0.05$ vs C17 EVs. (C) EECs were treated without (control) or with P17 EVs (10 $\mu \mathrm{g})$ for 48 h. Cell lysates were subjected to Western blotting. The bar graphs show acetylated NF-kB p65 levels normalized by total NF-kB p65 levels ( $n=3$ each). ${ }^{*} P<0.05$ vs control. (D) EECs were treated without or with P17 EVs $(10 \mu \mathrm{g})$ and/or TCPA1 $(20 \mathrm{nM}, \mathrm{NF}-\mathrm{kB}$ pathway inhibitor) for $48 \mathrm{~h}$. RNAs were subjected to qPCR to examine changes in TNF signaling pathway-related gene expression. ACTB and GAPDH mRNAs served as internal controls for RNA integrity. Letters over the bars indicate significant differences between treatment means $(P<0.05)$.

Cheng et al. 2017). In this study, down-regulation of PLAC8 was observed in C17 EVs-treated EECs, which may be caused by the autocrine effects of $\mathrm{C} 17 \mathrm{EVs}$. A previous study in which EVs are secreted from uterine epithelia as well as conceptus trophectoderm in ruminants (Burns et al. 2016) supports this finding. These results support that EVs, released from uterine endometrium and/or elongated conceptus, are involved in intercellular communications in an autocrine and/or paracrine manner.

In previous studies, transcriptome profiles, obtained from bovine endometrial explants treated with or without ovine IFNT, Al-derived conceptus, and
IVF-derived conceptuses, also demonstrated IFNTindependent endometrial response (Mathew et al. 2019, Sánchez et al. 2019). Among IFNT-independent DEGs found in those endometrial explants, only BIRC3 and ECM1 were found as common transcripts between theirs and our DEGs of P17 EVs-treated EECs. Finding only two transcripts as the common transcripts was not surprising because the experimental conditions of the previous studies and ours such as co-cultured time, the origin of IFNT, or the day of pregnant stage differed.

It is known that CD40 is expressed in a wide range of immune cells including monocytes, dendritic cells, and B cells and that upon CD40L binding to CD40, elicitation of immune and inflammatory processes is mediated by the activation of an intracellular NF-kB pathway signaling (Henn et al. 1998, Qian et al. 2002). In the present study, CD40L and its receptor CD40 was found in P17 EVs and EECs treated with P17 EVs, respectively. Treatment with $\mathrm{P} 17 \mathrm{EVs}$ induced the activation of NF-kB, and treatment with NF-kB signaling inhibitor down-regulated P17 EVs-induce TNF signaling pathway-related gene expression in EECs. In addition, our previous study indicated that higher expression of CD40 was found in EECs treated with intrauterine P17 EVs compared with those of intrauterine EVs on day 20 of pregnancy (unpublished observation). These findings suggest that P17 EVs containing CD40L could bind to CD40 displayed by endometrial epithelium, as well as immune cells, which induces inflammatory response mediated by the activation of NF-kB in endometrial epithelium during pre-attachment period.

A delicate interplay of pro- and anti-inflammatory signaling takes place within the endometrium during early pregnancy in mammals (Mor et al. 2011). Our work provides evidence that P17 EVs, independent of IFNT, induced significantly enriched 'TNF signaling pathway' and 'Inflammatory response' in EECs. During initial crosstalk with the endometrium, conceptuses released cytokines, many of which were characterized as pro-inflammatory mediators (Kelly et al. 2001, Mathew et al. 2016). The pro-inflammatory response enhances the expression of other cytokines and/or chemokines, which in turn recruit immune cells such as macrophages and dendritic cells to the site of inflammation (Granot et al. 2012). These immune cells secrete different factors that may affect uterine natural killer cell differentiation and stimulate the luminal endometrial cells to produce adhesion molecules enabling the attachment of conceptus to the endometrium. In humans, the success of implantation is apparently attributed to an injury-induced inflammatory response that leads to the development of an adequate decidua supporting implantation (Gnainsky et al. 2010). Taken together, these findings suggest that the inflammatory response in the endometrium induced by P17 EVs is required for the acquisition of uterine receptivity for successful implantation and the subsequent pregnancy outcome. 
In conclusion, the comparison of global transcriptome analyses among C17 EVs-, P17 EVs-, and IFNT-treated EECs revealed that induction of TNF signaling pathwayrelated genes via CD40L/CD40/NF-kB pathway was independent of IFNT effects in P17 EVs-treated EECs. Therefore, P17 EVs independent of IFNT, some of which secreted from conceptus, could induce a pro-inflammatory response in the endometrium to regulate the uterine receptivity, facilitating conceptus implantation to the uterine epithelium.

\section{Declaration of interest}

The authors declare that there is no conflict of interest that could be perceived as prejudicing the impartiality of the research reported.

\section{Funding}

This work was supported by Early-Career Scientists (18K14566 to K K) and Grant-in-Aid for Scientific Research (A) (16H02584 to $\mathrm{K}$ I) from Japan Society for the Promotion of Science.

\section{Author contribution statement}

$\mathrm{K} \mathrm{N}$ and $\mathrm{K} \mathrm{K}$ performed experiments and analyses. $\mathrm{K} \mathrm{N}, \mathrm{K} \mathrm{K}$, and $\mathrm{K} \mathrm{I}$ wrote the main manuscript text, and $\mathrm{K} \mathrm{N}$ and $\mathrm{K} \mathrm{K}$ prepared all figures and tables. A I and $\mathrm{M} \mathrm{H}$ were involved in the planning of the entire experimentation and provided uterine flushing and bovine tissues samples. $\mathrm{K} \mathrm{N}, \mathrm{K} \mathrm{K}, \mathrm{A} \mathrm{I}, \mathrm{K} \mathrm{I}$, and $\mathrm{MH}$ reviewed the manuscript.

\section{Acknowledgements}

The authors would like to thank Dr Koji Kimura, Graduate School of Environmental and Life Science, Okayama University, for bovine endometrial epithelial cells and Dr Michael Rupp, Tokai University-Kumamoto, for English editing. Computations were partially performed on the NIG supercomputer at ROIS National Institute of Genetics.

\section{References}

Bai R, Latifi Z, Kusama K, Nakamura K, Shimada M \& Imakawa K 2018 Induction of immune-related gene expression by seminal exosomes in the porcine endometrium. Biochemical and Biophysical Research Communications 495 1094-1101. (https://doi.org/10.1016/j. bbrc.2017.11.100)

Bauersachs S, Ulbrich SE, Reichenbach HD, Reichenbach M, Büttner M, Meyer HH, Spencer TE, Minten M, Sax G, Winter G et al. 2012 Comparison of the effects of early pregnancy with human interferon, alpha 2 (IFNA2), on gene expression in bovine endometrium. Biology of Reproduction 86 46. (https://doi.org/10.1095/biolreprod.111.094771)

Bazer FW \& Thatcher WW 2017 Chronicling the discovery of interferon tau. Reproduction 154 F11-F20. (https://doi.org/10.1530/REP-170257)

Burns G, Brooks K, Wildung M, Navakanitworakul R, Christenson LK \& Spencer TE 2014 Extracellular vesicles in luminal fluid of the ovine uterus. PLOS ONE 9 e90913. (https://doi.org/10.1371/journal. pone.0090913)
Burns GW, Brooks KE \& Spencer TE 2016 Extracellular vesicles originate from the conceptus and uterus during early pregnancy in sheep. Biology of Reproduction 94 56. (https://doi.org/10.1095/biolreprod.115.134973)

Burns GW, Brooks KE, O’Neil EV, Hagen DE, Behura SK \& Spencer TE 2018 Progesterone effects on extracellular vesicles in the sheep uterus. Biology of Reproduction 98 612-622. (https://doi.org/10.1093/biolre/ioy011)

Cheng Z, Chauhan L, Barry AT, Abudureyimu A, Oguejiofor CF, Chen X \& Wathes DC 2017 Acute bovine viral diarrhea virus infection inhibits expression of interferon tau-stimulated genes in bovine endometrium. Biology of Reproduction 96 1142-1153. (https://doi.org/10.1093/biolre/ iox056)

Cleys ER, Halleran JL, McWhorter E, Hergenreder J, Enriquez VA, da Silveira JC, Bruemmer JE, Winger QA \& Bouma GJ 2014 Identification of microRNAs in exosomes isolated from serum and umbilical cord blood, as well as placentomes of gestational day 90 pregnant sheep. Molecular Reproduction and Development 81 983-993. (https://doi. org/10.1002/mrd.22420)

Ealy AD \& Wooldridge LK 2017 The evolution of interferon-tau. Reproduction 154 F1-F10. (https://doi.org/10.1530/REP-17-0292)

Forde N \& Lonergan P 2017 Interferon-tau and fertility in ruminants. Reproduction 154 F33-F43. (https://doi.org/10.1530/REP-17-0432)

Forde N, Carter F, Spencer TE, Bazer FW, Sandra O, Mansouri-Attia N, Okumu LA, McGettigan PA, Mehta JP, McBride R et al. 2011 Conceptusinduced changes in the endometrial transcriptome: how soon does the cow know she is pregnant? Biology of Reproduction 85 144-156. (https://doi.org/10.1095/biolreprod.110.090019)

Forde N, McGettigan PA, Mehta JP, O'Hara L, Mamo S, Bazer FW, Spencer TE \& Lonergan P 2014 Proteomic analysis of uterine fluid during the pre-implantation period of pregnancy in cattle. Reproduction 147 575-587. (https://doi.org/10.1530/REP-13-0010)

Forde N, Bazer FW, Spencer TE \& Lonergan P 2015 'Conceptualizing' the endometrium: identification of conceptus-derived proteins during early pregnancy in cattle. Biology of Reproduction 92 156. (https://doi. org/10.1095/biolreprod.115.129296)

Gnainsky Y, Granot I, Aldo PB, Barash A, Or Y, Schechtman E, Mor G \& Dekel N 2010 Local injury of the endometrium induces an inflammatory response that promotes successful implantation. Fertility and Sterility $\mathbf{9 4}$ 2030-2036. (https://doi.org/10.1016/j.fertnstert.2010.02.022)

Granot I, Gnainsky Y \& Dekel N 2012 Endometrial inflammation and effect on implantation improvement and pregnancy outcome. Reproduction 144 661-668. (https://doi.org/10.1530/REP-12-0217)

Henn V, Slupsky JR, Gräfe M, Anagnostopoulos I, Förster R, MüllerBerghaus G \& Kroczek RA 1998 CD40 ligand on activated platelets triggers an inflammatory reaction of endothelial cells. Nature 391 591-594. (https://doi.org/10.1038/35393)

Hostager BS \& Bishop GA 2013 CD40-mediated activation of the NFкB2 pathway. Frontiers in Immunology 4 376. (https://doi.org/10.3389/ fimmu.2013.00376)

Ideta A, Urakawa M, Aoyagi Y \& Saeki K 2007 Early development in utero of bovine nuclear transfer embryos using early G1 and G0 phase cells. Cloning and Stem Cells 9 571-580. (https://doi.org/10.1089/ clo.2007.0017)

Kelly RW, King AE \& Critchley HO 2001 Cytokine control in human endometrium. Reproduction 121 3-19. (https://doi.org/10.1530/ rep.0.1210003)

Kusama K, Bai R, Ideta A, Aoyagi Y, Okuda K \& Imakawa K 2016 Regulation of epithelial to mesenchymal transition in bovine conceptuses through the interaction between follistatin and activin A. Molecular and Cellular Endocrinology 434 81-92. (https://doi. org/10.1016/j.mce.2016.06.017)

Kusama K, Bai R, Nakamura K, Okada S, Yasuda J \& Imakawa K 2017 Endometrial factors similarly induced by IFNT2 and IFNTC1 through transcription factor FOXS1. PLOS ONE 12 e0171858. (https://doi. org/10.1371/journal.pone.0171858)

Kusama K, Nakamura K, Bai R, Nagaoka K, Sakurai T \& Imakawa K 2018a Intrauterine exosomes are required for bovine conceptus implantation. Biochemical and Biophysical Research Communications 495 1370-1375. (https://doi.org/10.1016/j.bbrc.2017.11.176)

Kusama K, Bai R \& Imakawa K 2018 b Regulation of human trophoblast cell syncytialization by transcription factors STAT5B and NR4A3. Journal of Cellular Biochemistry 119 4918-4927. (https://doi.org/10.1002/ jcb.26721) 
Mamo S, Mehta JP, McGettigan P, Fair T, Spencer TE, Bazer FW \& Lonergan P 2011 RNA sequencing reveals novel gene clusters in bovine conceptuses associated with maternal recognition of pregnancy and implantation. Biology of Reproduction 85 1143-1151. (https://doi. org/10.1095/biolreprod.111.092643)

Mansouri-Attia N, Aubert J, Reinaud P, Giraud-Delville C, Taghouti G, Galio L, Everts RE, Degrelle S, Richard C, Hue I et al. 2009 Gene expression profiles of bovine caruncular and intercaruncular endometrium at implantation. Physiological Genomics 39 14-27. (https://doi.org/10.1152/physiolgenomics.90404.2008)

Mathew DJ, Lucy MC \& D Geisert R 2016 Interleukins, interferons, and establishment of pregnancy in pigs. Reproduction 151 111-122. (https:// doi.org/10.1530/REP-16-0047)

Mathew DJ, Sánchez JM, Passaro C, Charpigny G, Behura SK, Spencer TE \& Lonergan P 2019 Interferon tau-dependent and independent effects of the bovine conceptus on the endometrial transcriptome. Biology of Reproduction 100 365-380. (https://doi.org/10.1093/biolre/ioy199)

Mellisho EA, Velásquez AE, Nuñez MJ, Cabezas JG, Cueto JA, Fader C, Castro FO \& Rodríguez-Álvarez L 2017 Identification and characteristics of extracellular vesicles from bovine blastocysts produced in vitro. PLOS ONE 12 e0178306. (https://doi.org/10.1371/journal.pone.0178306)

Mor G, Cardenas I, Abrahams V \& Guller S 2011 Inflammation and pregnancy: the role of the immune system at the implantation site. Annals of the New York Academy of Sciences 1221 80-87. (https://doi. org/10.1111/j.1749-6632.2010.05938.x)

Nakamura K, Kusama K, Bai R, Sakurai T, Isuzugawa K, Godkin JD, Suda Y \& Imakawa K 2016 Induction of IFNT-stimulated genes by conceptus-derived exosomes during the attachment period. PLOS ONE 11 e0158278. (https://doi.org/10.1371/journal.pone.0158278)

Nakamura K, Kusama K, Bai R, Ishikawa S, Fukushima S, Suda Y \& Imakawa K 2017 Increase in complement iC3b is associated with antiinflammatory cytokine expression during late pregnancy in mice. PLoS ONE 12 e0178442. (https://doi.org/10.1371/journal.pone.0178442)

Nguyen HP, Simpson RJ, Salamonsen LA \& Greening DW 2016 Extracellular vesicles in the intrauterine environment: challenges and potential functions. Biology of Reproduction 95 109. (https://doi.org/10.1095/ biolreprod.116.143503)

Piehl LL, Fischman ML, Hellman U, Cisale H \& Miranda PV 2013 Boar seminal plasma exosomes: effect on sperm function and protein identification by sequencing. Theriogenology 79 1071-1082. (https:// doi.org/10.1016/j.theriogenology.2013.01.028)

Prunotto M, Farina A, Lane L, Pernin A, Schifferli J, Hochstrasser DF, Lescuyer P \& Moll S 2013 Proteomic analysis of podocyte exosomeenriched fraction from normal human urine. Journal of Proteomics 82 193-229. (https://doi.org/10.1016/j.jprot.2013.01.012)

Qian Y, Zhao Z, Jiang Z \& Li X 2002 Role of NF kappa B activator Act1 in CD40-mediated signaling in epithelial cells. PNAS 99 9386-9391. (https://doi.org/10.1073/pnas.142294499)

Roberts RM 201730 years on from the molecular cloning of interferon-tau. Reproduction 154 E1-E2. (https://doi.org/10.1530/REP-17-0585)

Ruiz-González I, Xu J, Wang X, Burghardt RC, Dunlap KA \& Bazer FW 2015 Exosomes, endogenous retroviruses and toll-like receptors: pregnancy recognition in ewes. Reproduction 149 281-291. (https://doi. org/10.1530/REP-14-0538)

Sakurai T, Bai H, Bai R, Arai M, Iwazawa M, Zhang J, Konno T, Godkin JD, Okuda K \& Imakawa K 2012 Coculture system that mimics in vivo attachment processes in bovine trophoblast cells. Biology of Reproduction 87 60. (https://doi.org/10.1095/biolreprod.112.100180)

Sánchez JM, Mathew DJ, Behura SK, Passaro C, Charpigny G, Butler ST, Spencer TE \& Lonergan P 2019 Bovine endometrium responds differentially to age-matched short and long conceptuses. Biology of Reproduction 101 26-39. (https://doi.org/10.1093/biolre/ioz060)

Simintiras CA, Sánchez JM, McDonald M, Martins T, Binelli M \& Lonergan P 2019a Biochemical characterization of progesterone-induced alterations in bovine uterine fluid amino acid and carbohydrate composition during the conceptus elongation window. Biology of Reproduction 100 672-685. (https://doi.org/10.1093/biolre/ioy234)

Simintiras CA, Sánchez JM, McDonald M \& Lonergan P 2019 b Progesterone alters the bovine uterine fluid lipidome during the period of elongation. Reproduction 157 399-411. (https://doi.org/10.1530/REP-18-0615)

Simintiras CA, Sánchez JM, McDonald M \& Lonergan P 2019C The influence of progesterone on bovine uterine fluid energy, nucleotide, vitamin, cofactor, peptide, and xenobiotic composition during the conceptus elongation-initiation window. Scientific Reports 97716. (https://doi.org/10.1038/s41598-019-44040-6)

Spencer TE, Forde N, Dorniak P, Hansen TR, Romero JJ \& Lonergan P 2013 Conceptus-derived prostaglandins regulate gene expression in the endometrium prior to pregnancy recognition in ruminants. Reproduction 146 377-387. (https://doi.org/10.1530/REP-13-0165)

Théry C, Witwer KW, Aikawa E, Alcaraz MJ, Anderson JD, Andriantsitohaina R, Antoniou A, Arab T, Archer F, Atkin-Smith GK et al. 2018 Minimal information for studies of extracellular vesicles 2018 (MISEV2018): a position statement of the International Society for extracellular vesicles and update of the MISEV2014 guidelines. Journal of Extracellular Vesicles 7 1535750. (https://doi.org/10.1080/20013078. 2018.1535750)

Walker CG, Meier S, Littlejohn MD, Lehnert K, Roche JR \& Mitchell MD 2010 Modulation of the maternal immune system by the pre-implantation embryo. BMC Genomics 11 474. (https://doi.org/10.1186/1471-216411-474)

Wiltbank MC, Baez GM, Garcia-Guerra A, Toledo MZ, Monteiro PL, Melo LF, Ochoa JC, Santos JE \& Sartori R 2016 Pivotal periods for pregnancy loss during the first trimester of gestation in lactating dairy cows. Theriogenology 86 239-253. (https://doi.org/10.1016/j. theriogenology.2016.04.037)

Received 16 July 2019

First decision 27 August 2019

Revised manuscript received 27 January 2020

Accepted 6 February 2020 